\title{
Frontal, Striatal, and Medial Temporal Sensitivity to Value Distinguishes Risk-Taking from Risk-Aversive Older Adults during Decision Making
}

\author{
ㄱoshua 0.S. Goh, ${ }^{1,2,3,4}$ Yu-Shiang Su, ${ }_{1}^{1}$ Yong-Jheng Tang, ${ }^{1}$ Anna C. McCarrey, ${ }^{4}$ Alexander Tereshchenko, ${ }^{4}$ \\ Wendy Elkins, ${ }^{4}$ and Susan M. Resnick ${ }^{4}$ \\ ${ }^{1}$ Graduate Institute of Brain and Mind Sciences, College of Medicine, National Taiwan University, Taipei, Taiwan 10051, ${ }^{2}$ Neurobiology and Cognitive \\ Science Center and ${ }^{3}$ Department of Psychology, National Taiwan University, Taipei, Taiwan 10617, and ${ }^{4}$ Laboratory of Behavioral Neuroscience, Intramural \\ Research Program, National Institute on Aging, Baltimore, Maryland 21224
}

Aging compromises the frontal, striatal, and medial temporal areas of the reward system, impeding accurate value representation and feedback processing critical for decision making. However, substantial variability characterizes age-related effects on the brain so that some older individuals evince clear neurocognitive declines whereas others are spared. Moreover, the functional correlates of normative individual differences in older-adult value-based decision making remain unclear. We performed a functional magnetic resonance imaging study in 173 human older adults during a lottery choice task in which costly to more desirable stakes were depicted using low to high expected values (EVs) of points. Across trials that varied in EVs, participants decided to accept or decline the offered stakes to maximize total accumulated points. We found that greater age was associated with less optimal decisions, accepting stakes when losses were likely and declining stakes when gains were likely, and was associated with increased frontal activity for costlier stakes. Critically, risk preferences varied substantially across older adults and neural sensitivity to EVs in the frontal, striatal, and medial temporal areas dissociated risk-aversive from risk-taking individuals. Specifically, risk-averters increased neural responses to increasing EVs as stakes became more desirable, whereas risk-takers increased neural responses with decreasing EV as stakes became more costly. Risk preference also modulated striatal responses during feedback with risk-takers showing more positive responses to gains compared with risk-averters. Our findings highlight the frontal, striatal, and medial temporal areas as key neural loci in which individual differences differentially affect value-based decision-making ability in older adults.

Key words: aging; decision making; fMRI; individual differences; older adults; reward system

\section{Significance Statement}

Frontal, striatal, and medial temporal functions implicated in value-based decision processing of rewards and costs undergo substantial age-related changes. However, age effects on brain function and cognition differ across individuals. How this normative variation relates to older-adult value-based decision making is unclear. We found that although the ability make optimal decisions declines with age, there is still much individual variability in how this deterioration occurs. Critically, whereas risk-averters showed increased neural activity to increasingly valuable stakes in frontal, striatal, and medial temporal areas, risk-takers instead increased activity as stakes became more costly. Such distinct functional decision-making processing in these brain regions across normative older adults may reflect individual differences in susceptibility to age-related brain changes associated with incipient cognitive impairment.

\section{Introduction}

Stimuli that predict reward evoke higher frontostriatal activity than stimuli that induce expectations of less reward or higher cost

\footnotetext{
Received April 27, 2016; revised 0ct. 21, 2016; accepted 0ct. 22, 2016.

Author contributions: J.O.S.G., A.T., and S.M.R. designed research; J.O.S.G., A.T., and W.E. performed research; J.O.S.G., Y.-S.S., Y.-J.T., and A.C.M. analyzed data; J.O.S.G., Y.-S.S., Y.-J.T., A.C.M., and S.M.R. wrote the paper.

This work was supported in part by the Intramural Research Program of the National Institute on Aging, National Institutes of Health; by the National Science Council, Taiwan, Grant \#102-2410-H-002-004; and by the Ministry of Science and Technology, Taiwan, Grant\#103-2410-H-002-082. We also thank Anne M. Farrell and Brian J. White for their conceptual input.

The authors declare no competing financial interests.
}

(Schultz et al., 1997; Breiter et al., 2001; Knutson et al., 2001; Preuschoff et al., 2006). Moreover, whereas expected outcomes minimally modulate frontostriatal activity relative to baseline, unexpected negative outcomes reduce activity (negative prediction error) in these regions, while unexpected positive outcomes

Correspondence should be addressed to Joshua 0. S. Goh, PhD, Graduate Institute of Brain and Mind Sciences, College of Medicine, National Taiwan University, No. 1 Ren-Ai Road, Section 1, RM 1554, Zhongzheng District, Taipei, Taiwan 10051.E-mail: joshuagoh@ntu.edu.tw

DOI:10.1523/JNEUROSCI.1386-16.2016

Copyright $\odot 2016$ the authors $\quad 0270-6474 / 16 / 3612498-12 \$ 15.00 / 0$ 
enhance it (positive prediction error). The fidelity of such Bayesian-like choice value prediction and feedback-based adjustment in these frontal, striatal, and medial temporal areas (Koster et al., 2015) is crucial for rapid and accurate assessment of environmental stochastic dangers and opportunities. Compromises to these areas can bias individuals to inappropriately engage in risk-aversive or risk-taking behaviors leading to suboptimal outcomes.

With aging, structural, neurobiological, and functional changes affect the frontal, striatal, and medial temporal areas and reduce the selectivity of neural responses to different stimuli (Goh and Park, 2009; Eppinger et al., 2011; Goh, 2011). Thus, neural representations of stimulus reward and cost may be less veridical in older than in younger individuals, culminating in suboptimal decisions. Indeed, older adults showed less neural sensitivity than younger adults to different levels of anticipated monetary losses (Samanez-Larkin et al., 2007). Greater age was also associated with greater variability of nucleus accumbens (NAcc) activity, possibly reflecting noisier value signaling related to greater risk taking (Samanez-Larkin et al., 2010).

However, there are substantial individual differences in the effects of aging on the brain and cognitive processing. Even within cognitively normal older adults, some individuals show greater age-related declines in executive processing and related frontal processes that are predictive of later mild cognitive impairment or Alzheimer's disease (Goh et al., 2012, 2013; BeasonHeld et al., 2013). In the Iowa Gambling Task, young adults show better reward learning than older adults as a group, but at least a subset of older adults show learning comparable to young adults (Denburg et al., 2005). Treatment with a dopamine agonist increased frontostriatal dopamine responsiveness that correlated with better reward learning in some older adults, whereas others were unaffected or even showed poorer learning (Chowdhury et al., 2013). Apart from cognitive and reward learning differences, individual variability in value representations during choice may also explain why some older adults engage in more risk-taking behaviors while others are more risk-averse. Nevertheless, the neural activities underlying individual differences in older-adult value representations remain unclear.

In this study, we characterize the functional neural correlates of value processing underlying the influences of age and individual differences that dissociate risk-averters from risk-takers in a large sample of cognitively normal older adults from the Baltimore Longitudinal Study of Aging (BLSA). Participants underwent a functional magnetic resonance imaging (fMRI) lottery choice task experiment in which they accepted or declined costly to more desirable stakes depicted by negative to positive expected values (EVs) over trials. We assessed acceptance rates of each participant's choices over these trials, which varied in value magnitude and probability, and indexed risk preference using the decision threshold (DT) EVs at which an individual switched from declining to accepting stakes (Dohmen et al., 2011). We expected greater age to correspond with suboptimal acceptance rates and altered functional responses to increasing EVs. In addition to age effects, we expected that neural representations of EVs in the frontal, striatal, and medial temporal areas of the reward system would vary across individuals such that risk-averters and risk-takers evince dissociated neural responses to stimulus values during choices that correlate with their decision behaviors. Specifically, risk-averters should show greater sensitivity to increasing EVs relative to more risk-neutral counterparts, whereas risk-takers, being more partial to experiencing gains under risk and less motivated by safe options,
Table 1. Demographic details of the final 173 participants who underwent the fMRI lottery choice task experiment ${ }^{a}$

\begin{tabular}{lllll}
\hline Variable & $N$ & Mean (SD) & Minimum & Maximum \\
\hline Age (years) & 173 & $71.4(11.0)$ & 43.0 & 90.7 \\
Years of education & 173 & $16.7(2.1)$ & 12 & 21 \\
MMSE & $137^{b}$ & $28.6(1.4)$ & 23 & 30 \\
Sex (male/female) & $78 / 95$ & - & - & - \\
Handedness (right/left) & $162 / 11$ & - & - & - \\
Race (white/African-American/others) & $123 / 40 / 10$ & - & - & - \\
\hline
\end{tabular}

${ }^{a}$ Performance in the MMSE was within normal range based on published, age-adjusted norms.

${ }^{b}$ Data were available only for participants $\geq 60$ years old because no one younger is administered the MMSE in the Baltimore Longitudinal Study of Aging.

should show greater sensitivity to decreasing EVs. Finally, we evaluated how risk preferences during choice also modulate reward processing during feedback. Individuals with more extreme risk preferences should evince reduced responses to unexpected loss outcomes ("disappointment") or enhanced responses to unexpected wins ("surprise").

\section{Materials and Methods}

Participants. Two hundred and twenty-eight participants underwent this cross-sectional fMRI lottery choice task experiment conducted from 2011 to 2013 as part of the ongoing neuroimaging substudy of the BLSA (Resnick et al., 2000). All participants were cognitively normal (no mild cognitive impairment or dementia) at the time of the fMRI experiment based on prior annual regular case conferences with consensus diagnosis using neurological assessments, and on clinical and neuropsychological data (Kawas et al., 2000; Driscoll et al., 2006). The local institutional review board approved the research protocol for this study, and written informed consent was obtained from all participants. Of these participants, 173 entered the final sample (Table 1) after these exclusions: the data of four participants were unavailable due to technical faults; eight participants had brain image artifacts; 10 participants had excessive head motion ( $>2 \mathrm{~mm}$ translation or $>2^{\circ}$ rotation); two participants experienced no losses in the lottery choice task throughout an entire run; one participant gave no responses to one condition; and 30 participants showed atypical decision behaviors, including accepting more low than high probability trials, or having DTs that were outliers based on Grubb's test, which call into question understanding of the task.

fMRI lottery choice task stimuli and procedure. EV was manipulated in a fMRI lottery choice task experiment (Fig. 1). There were 72 lottery trials, each with a choice phase and a feedback phase. For the choice phase, the top text depicted the magnitude of points at stake $(M)$, and the bottom texts depicted the percentage probability of winning $(P)$ or losing $(1-P)$ the stakes. $M$ and $P$ comprised the EV across trials according to the equation $\mathrm{EV}=P M+(1-P)(-M)$. Higher positive EVs denote more desirable stakes, for example when a trial has high $M$ and high $P$ (close to 1). By contrast, lower negative EVs denote more costly stakes, for example when a trial has high $M$ but low $P$ (close to 0 ). We applied six discrete combination choice conditions of ranges of $P$ and $M$ (HH: high $P$, high $M$; HL: high $P$, low $M$; MH: middle $P$, high $M$; ML: middle $P$, low $M$; LH: low $P$, high $M$; LL: low $P$, low $M$; 12 trials per condition; Table 2) to facilitate pseudorandom ordering of trial EVs as well as predetermined outcomes (see below). Specifically, trials were distributed in the eventrelated fMRI experiment such that $\leq 3$ in a row had the same choice condition or predetermined outcome. Two lists of stimuli with different $M$ and $P$ but similar ranges of EVs were generated for counterbalancing across participants (91 saw list A; 82 saw list B) to minimize list-specific effects. All trials were unique so that no number combinations of $P$ and $M$ were repeated throughout the study.

For the feedback phase, the top text depicted the outcome points for that trial and the bottom number showed the current accumulated points. There were three feedback conditions: gain, loss, or null (decline to respond or null response). Outcomes were stochastically predetermined based on the given choice probabilities with the restriction that no choice condition predetermined outcomes could have only winning or 


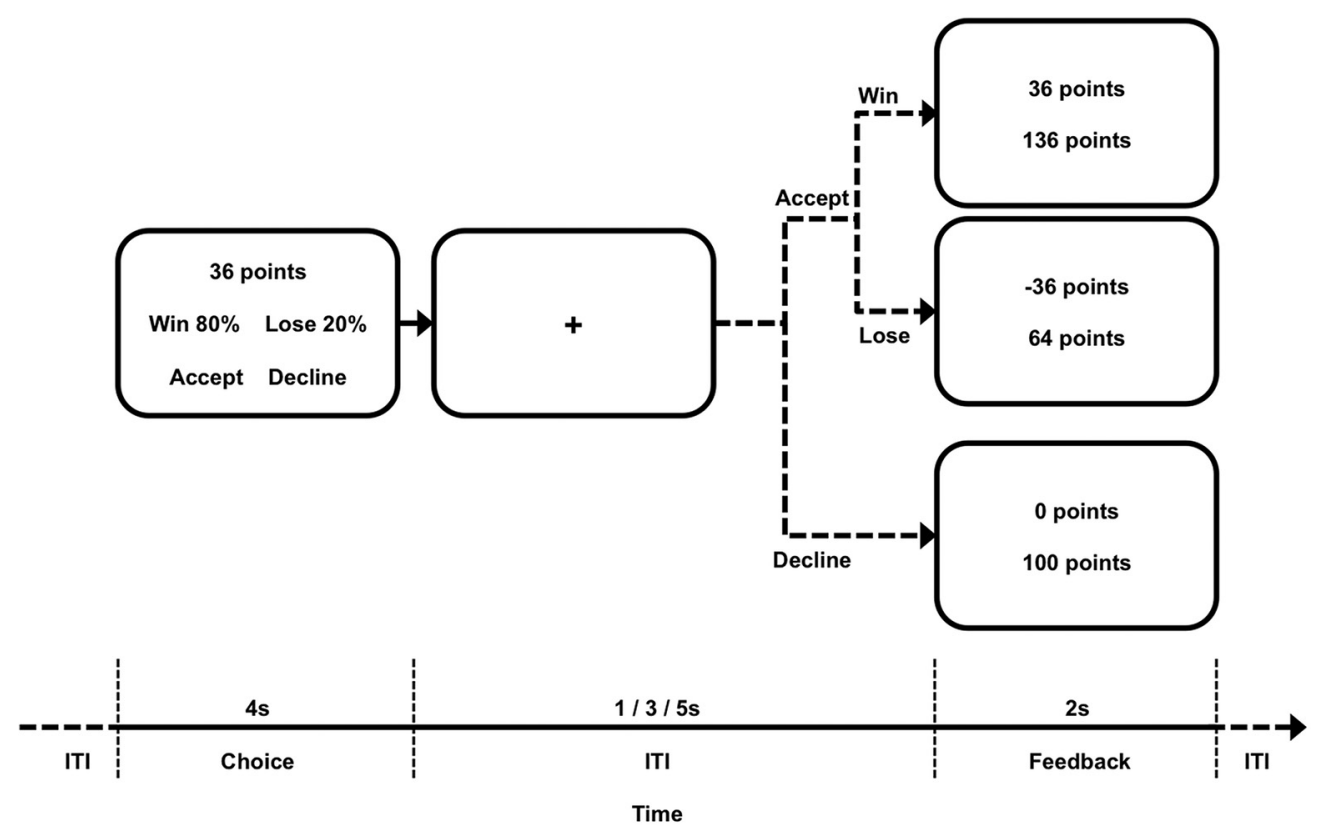

Figure 1. Sample trial in the fMRI lottery choice task experiment. During the choice phase of each trial, participants were shown the magnitude of points at stake along with the probability of winning and losing the stakes. Participants then decided to accept or decline the offer within $4 \mathrm{~s}$. During the feedback phase, participants were shown the outcome (top number) and accumulated points (bottom number). No change of points occurred if participants declined. Probability and magnitude of points during the choice phase were manipulated to compose various levels of negative to positive EVs over trials. Fixations were shown during variable intertrial intervals (ITI).

Table 2. Ranges of win probabilities $(P)$, magnitudes $(M)$, expected values (EV), and percentage of actual winning trials (stochastically predetermined based on trial $P$ ) in the lottery choice task

\begin{tabular}{llcccl}
\hline List & Condition & $P(\%)$ & $M$ (points) & EV (points) & Win trials (\%) \\
\hline A & & & & & \\
& HH & $69-88$ & $26-33$ & $9.9-25.1$ & 83 \\
& HL & $69-88$ & $2-9$ & $0.8-6.8$ & 75 \\
& MH & $47-53$ & $26-33$ & -2.0 to 2.0 & 58 \\
& ML & $47-53$ & $2-9$ & -0.5 to 0.5 & 33 \\
& LH & $12-31$ & $26-33$ & -25.1 to -9.9 & 17 \\
& LL & $12-31$ & $2-9$ & -6.8 to -0.8 & 25 \\
B & & & & & \\
& HH & $65-93$ & $27-36$ & $8.1-31.0$ & 75 \\
& HL & $65-93$ & $3-12$ & $0.9-10.3$ & 75 \\
& MH & $46-52$ & $27-36$ & -1.4 to 2.9 & 42 \\
& ML & $46-52$ & $3-12$ & -0.5 to 1.0 & 58 \\
& LH & $7-35$ & $27-36$ & -31.0 to -8.1 & 25 \\
& LL & $7-35$ & $3-12$ & -10.3 to -0.9 & 25 \\
\hline
\end{tabular}

only losing trials (Table 2). Note that this resulted in choice conditions in which the actual number of win trials did not correspond to the range of probabilities stated in the stimuli. Nevertheless, the discretization of trials into six conditions was internal to our analysis and participants were only instructed to make decisions based on trial-wise information.

Over two functional runs in the scanner (each $360 \mathrm{~s}$ duration), choice and feedback stimuli were presented, interleaved by fixation crosses with jittered intertrial intervals. Twenty second fixation intervals preceded and ended each run to facilitate baseline signal estimation. Participants were instructed to accumulate as many points as possible by deciding during the choice phase of each trial whether to accept or decline the given stakes and indicate their decision with assigned button presses. Choice stimuli remained on screen for a full $4 \mathrm{~s}$ even after responses. For accepted trials, feedback was either a gain or a loss, based on the predetermined outcomes. For declined trials, a null feedback was given. Trials in which participants took $>4 \mathrm{~s}$ to respond also resulted in null feedback plus a textual reminder to respond. Participants were trained with a practice version of the task before scanning to ensure that they understood their task goal and were familiar with the range of probabilities and points magnitudes used. Stimuli were presented as black text on white background using E-Prime software (Psychology Software Tools) via back-projection onto a screen in the scanner room, which participants viewed through a mirror mounted on the head coil. All participants were able to read the stimuli either without aid or with corrected vision.

Brain imaging protocol. Brain imaging data were acquired in a $3 \mathrm{~T}$ Philips Achieva system (Philips Healthcare) with an eight-channel head coil. For each participant, 180 functional scans were acquired in each run using an echo-planar imaging sequence with 37 axial slices parallel to the anterior-posterior commissural plane [voxel size, $3.00 \times 3.00 \times 3.00$ $\mathrm{mm}$; in-plane matrix size, $80 \times 72$; repetition time $(\mathrm{TR})=2 \mathrm{~s}$; echo time $(\mathrm{TE})=30 \mathrm{~ms}$; flip-angle, $75^{\circ}$ ]. A high-resolution T2 image coplanar to the functional scans was also acquired for coregistration, with 50 slices (voxel size, $0.94 \times 0.96 \times 3.00 \mathrm{~mm}$; in-plane matrix size, $256 \times 218$; $\mathrm{TR}=3 \mathrm{~s} ; \mathrm{TE}=100 \mathrm{~ms}$; flip-angle, $90^{\circ}$ ). Finally, a high-resolution $\mathrm{T} 1$ magnetization prepared rapid acquisition gradient echo image was acquired for normalization to standard space with 170 sagittal slices (voxel size, $1.20 \times 1.00 \times 1.00 \mathrm{~mm}$; in-plane matrix size, $256 \times 240 \mathrm{~mm}$; $\mathrm{TR}=$ $6.5 \mathrm{~ms}$; TE $=3.1 \mathrm{~ms}$; flip angle, $8^{\circ}$ ).

Lottery choice task behavioral analysis. Behavioral analyses were conducted using R version 3.0.2 (www.r-project.org). Since the safe outcome in all trials would always decline with no gain or loss (zero EV), individual indices of risk preference were estimated as the DT that denotes the certainty equivalent (Dohmen et al., 2011) of zero EV as follows. For each participant, logistic regression was first applied on the binary behavioral responses (accept, 1; decline, 0 ; null response trials excluded) as the dependent variable over the range of negative to positive EVs as the independent variable across trials using the model $\log ($ Response $)=\beta_{0}+$ $\beta_{1} \mathrm{EV}+\varepsilon$. The DT was then computed as $-\left(\beta_{0} / \beta_{1}\right)$, which is the estimated EV when the participant's likelihood of accepting is 0.5 . In our experiment, a risk-neutral agent would have a DT (or certainty equivalent) of 0 , which is exactly the $\mathrm{EV}$ when the probability of winning or losing is 0.5 . Higher to lower DTs then indicate certainty equivalents greater or lower than $\mathrm{EV}=0$ when probability of winning or losing was 0.5 , reflecting risk-averse to risk-taking preferences, respectively. To visualize continuous associations between risk preference and behavioral and brain responses, modeled data in respective analyses were plotted over four incremental levels of DT. These levels were set as the mean DTs of the lower $[\overline{\mathrm{DT}}(\mathrm{SD})=-6.6(3.7) ; N=60]$ and upper $[\overline{\mathrm{DT}}(\mathrm{SD})=$ 
$-0.7(0.7) ; N=61]$ halves of participants with DT $\leq 0$ for very risktaking (VRT) and moderately risk-taking (MRT) levels, respectively, and the lower $[\overline{\mathrm{DT}}(\mathrm{SD})=0.3(0.3) ; N=26]$ and upper $[\overline{\mathrm{DT}}(\mathrm{SD})=2.8(2.4)$; $N=26]$ halves of participants with DT $>0$ for moderately risk-averse (MRA) and very risk-averse (VRA) levels, respectively.

Mean acceptance rates and response times for each of the six discrete conditions were obtained for each participant. However, to formally validate the effects of EV, age, and individual risk preferences (indexed by DT) in modulating lottery choice task behavioral performance, grouplevel mixed-model analyses were computed for the trial-wise acceptance rates and response time data. Specifically, for acceptance rates over the range of EVs used, a logistic mixed model was implemented with binary responses from all trials of all participants as the dependent variable and trial-wise EV, age, DT, and the interactions of EV with age and EV with $\mathrm{DT}$ as fixed effects of interest. Trial-wise variances (computed as $P[M-$ $\left.\mathrm{EV}]^{2}+[1-P][(-\mathrm{M}) \text { minus } \mathrm{EV}]^{2}\right)$ may also modulate uncertainty about trial-wise EVs in relation to risk processing (Preuschoff et al., 2006) and were included as a fixed-effect covariate. Other fixed-effect covariates were current accumulated scores and sex, and participant was a random effect. We then specifically tested the significances of regression coefficients for the effects of EV, age, DT, and the specified interactions based on this logistic mixed model. A similar analysis was applied to the response time data for all trials except that a linear mixed model was used and EV was modeled to the quadratic term, based on inspection of trends in the data.

fMRI data preprocessing. Analyses of functional brain data were conducted using SPM8 (Statistical Parametric Mapping, Wellcome Trust Centre for Neuroimaging, UK). For each participant, functional images were preprocessed with motion realignment and slice-time correction. $\mathrm{T} 2$ anatomical images were coregistered to the functional images, then $\mathrm{T} 1$ images to the coregistered T2 images. T2 images for five participants were unavailable so that coregistration of functional to T1 images were applied directly for these cases. Functional images were then normalized to a study-specific template generated from participants' coregistered T1 images using diffeomorphic anatomical registration through exponentiated linear algebra (Ashburner, 2007). Normalized functional images were then affine-transformed to the standard Montreal Neurological Institute template space and smoothed with a 3D $8 \mathrm{~mm}$ Gaussian kernel.

fMRI analysis of neural responses during choice and feedback. We applied a standard first-level analysis on the preprocessed functional images to estimate mean neural responses for each of the six discrete conditions for each participant. This involved a general linear model that included delta function onset regressors (duration, $0 \mathrm{~s}$ for this event-related design) for each of the six choice conditions (HH, HL, MH, ML, LH, LL) and each of the three feedback conditions (gain, loss, null), which were then convolved with the hemodynamic response function (HRF). Covariates in this model included one regressor for trial-wise variances and one for current accumulated scores, which were generated by convolving the HRF with a choice onset regressor modulated by variances and current scores (with no orthogonalization), respectively. Six movement parameters were also included as head-motion covariates, resulting in a total of 17 regressors in this model.

For formal testing of age and individual differences in neural processing in the lottery choice task, we sought to identify brain regions that linearly changed neural responses with trial-wise EV as well as regions in which risk preferences modulated these linear response changes. We reasoned that linear responses reflect neural activity that increased (or decreased) with increasing value, at least in terms of rank order. Nonlinear responses that may reflect transitivity violations or distortions are not presently evaluated in this study. Note that we excluded participants with behavioral performances that had these counter-indications (see above). Estimates of each participant's neural sensitivity to EV during the choice phase were obtained by submitting the preprocessed functional images to a model-based first-level analysis. This model included one regressor for choice onsets, one for trial-wise EV (during choice), and one for each of the three feedback conditions (gain, loss, null). There was also one covariate regressor for trial-wise variances, one for current accumulated scores, and six head-movement parameters resulting in a total of 13 regressors in this model. Regressors for choice onset and feedback were delta function onsets convolved with the HRF only. Trial-wise EV, variance, and current score regressors were based on the HRF-convolved choice-onset regressor modulated by the respective trial-wise variables (with no orthogonalization). This analysis resulted in whole-brain voxelwise estimates of each participant's neural sensitivity to EV, controlling for the other variables, with higher positive voxel coefficients indexing greater sensitivity to increasingly desirable EVs and more negative voxel coefficients indexing greater sensitivity to increasingly costly EVs. These individual whole-brain EV estimates were then passed into a secondlevel group analysis using age and risk preference (DT) as independent variables, with sex as a covariate. The intercept of this second-level analysis yields brain areas in which trial-wise EV modulated neural activity at the group level after accounting for age and individual differences in risk preference. The effect of age in the model reveals brain areas in which neural sensitivity to trial-wise EV variation differed from younger to older adults (ranging from 43 to 91 years), accounting for individual differences in risk preference and the group mean response to EV. Finally, the effect of DT reflects brain areas in which individual risk preference modulated neural EV sensitivity after accounting for age and the group mean response to EV. Tests for significant contrasts were based on a voxel threshold of $p<0.001$ (uncorrected) with cluster extent correction of $>20$ voxels, except for the striatal and medial temporal regions. For these smaller structures, we created a subcortical volume mask that included the caudate, putamen, globus pallidus, thalamus, parahippocampal, hippocampal, and amygdala regions (based on the Automatic Anatomical Labeling atlas; Tzourio-Mazoyer et al., 2002). Within this mask, we adopted a small volume correction approach and evaluated voxels surpassing $p<0.005$ (uncorrected) with cluster extent correction of $>15$. Both criteria are within recommended standards to relate cognitive variables to brain functional measures (Lieberman and Cunningham, 2009) and also comparable to those used in previous studies examining value-based decision processing in these regions (SamanezLarkin et al., 2007). Both of these significance criteria also fulfilled a whole-brain $\alpha$ of $p<0.05$ using Monte Carlo simulation with 10,000 iterations (Slotnick et al., 2003, 2011).

Regions-of-interest (ROIs) for further analyses were defined as contiguously significant voxels within spheres of $10 \mathrm{~mm}$ radius around peak contrast voxels. To interrogate ROI EV-related responses during the choice phase in greater detail, the response estimates for choice onset and trial-wise EV from each participant's model-based first-level analysis were extracted. These individual choice and EV response estimates were then grouped based on the four levels of risk preference. For each risk preference level, the mean choice and mean EV response estimates across individuals in that group were then used to derive the modeled linear neural responses over the range of EVs in the task. Estimated responses to the six choice conditions from the standard first-level analysis were also extracted from ROIs to aid visual comparison of measured to modeled results.

Feedback response estimates from the first-level model-based analysis were further used to perform a conjunction analysis for voxels that simultaneously showed significant positive gain response and significant negative loss response relative to baseline fixation with a threshold of $p<$ 0.001 (cluster size, $>20$ ). This conjunction was applied to identify brain areas involved in canonical prediction errors during feedback, which we defined as regions that generated both positive signals for rewards and negative signals for punishments. Gain and loss responses were then extracted from ROIs identified based on the conjunction analysis. These were then passed into linear models with DT, its quadratic term, and age as independent variables, and sex as a covariate. We then examined statistical significance of the regression coefficients associated with DT in the models to evaluate how risk preference modulated responses to gain and loss outcomes during feedback.

\section{Results}

Individual differences in lottery choice task behavioral performance

Across participants, the distribution of DTs was right-skewed with mean $<0$, indicating a general risk-taking behavioral pref- 
erence in our sample of older adults $[\overline{\mathrm{DT}}$ $(\mathrm{SD})=-2.07$ (4.21), $t_{(172)}=-6.47, p<$ 0.001; Fig. 2]. Applying a linear regression model with DT as the dependent variable and age (mean-centered) as the independent variable with sex as a covariate, yielded no further effect of greater age in this older-adult sample in modulating the overall risk-taking preference. Critically, there were substantial individual differences in risk preferences reflected by the range of negative to positive DTs (minimum, -16.4 ; median, -0.80 ; maximum, 10.9] with 52 of 173 individuals showing $\mathrm{DT}>0$.

Consistent with reduced neural sensitivity to different values, greater age was associated with more suboptimal decision behavior characterized by higher acceptance rates at more costly negative EVs and lower acceptance rates at more desirable positive EVs (Fig. $3 A, D$ ). Moreover, acceptance rates increased as EVs increased and also as risk taking increased (VRA $<$ MRA $<$ MRT $<$ VRT), particularly for more costly negative EVs (Fig. $3 B, E)$. A logistic mixed model at the group level formally validated how age and risk preference modulated acceptance rates over the range of EVs presented in the lottery choice task (see Materials and Methods). Log odds of acceptance rates increased with increasing $\mathrm{EV}$ (EV: $Z=39.7, p<0.001$ ), decreased with greater age (age: $Z=-2.62, p<0.01$ ), and decreased with higher risk-aversion (DT: $Z=-14.0, p<0.001$ ). Importantly, the effect of EV on acceptance rates was also modulated by age $\left(\mathrm{EV}^{\star}\right.$ age: $Z=-5.90$, $p<0.001)$ and risk preference $\left(\mathrm{EV}^{\star} \mathrm{DT}: Z=14.6\right.$, $p<0.001)$. These interactions were due to decreasing influence of EVs on participant decisions as age or risk taking increased.

Response time model analysis showed a significant convex quadratic overall effect of EVs such that response time was estimated to be slowest at $\mathrm{EV}=-12.6$ on average, becoming faster with more extreme $\mathrm{EVs}\left(\mathrm{EV}^{2}: t_{(11860)}=-15.0, p<\right.$ 0.001 ; Fig. $3 C, F)$. Although there was no significant direct effect of risk preference, the convex quadratic effect of EVs on response time was reduced with increasing risk taking $\left(\mathrm{EV}^{2 \star} \mathrm{DT}: t_{(11860)}=-4.05, p<0.001 ; \mathrm{EV}^{\star} \mathrm{DT}: t_{(11850)}=7.93\right.$, $p<0.001)$ and approached a more linear trend with slower response times as EVs decreased $\left(\mathrm{EV}: t_{(11860)}=-13.0, p<\right.$ $0.001)$. Greater age was associated with an overall slowing of response times (age: $t_{(177)}=2.86, p<0.01$ ), which did not interact with $\mathrm{EV}$ or risk preference.

We considered that individual risk preferences might reflect differences in cognitive ability as well. To examine this, we further applied a linear model with DT (up to the quadratic term) as a variable explaining Mini-Mental State Examination (MMSE) scores with age and sex as covariates. Significant negative effects of DT $\left(t_{(132)}=-2.67, p<0.01\right)$ and $\mathrm{DT}^{2}$ $\left(t_{(132)}=-2.15, p<0.05\right)$ indicated a convex quadratic relationship between risk preference and MMSE performance (Fig. 2). Specifically, MMSE score was estimated to be maxiMRA, to VRA.
Distribution of Decision Threshold $(n=173)$
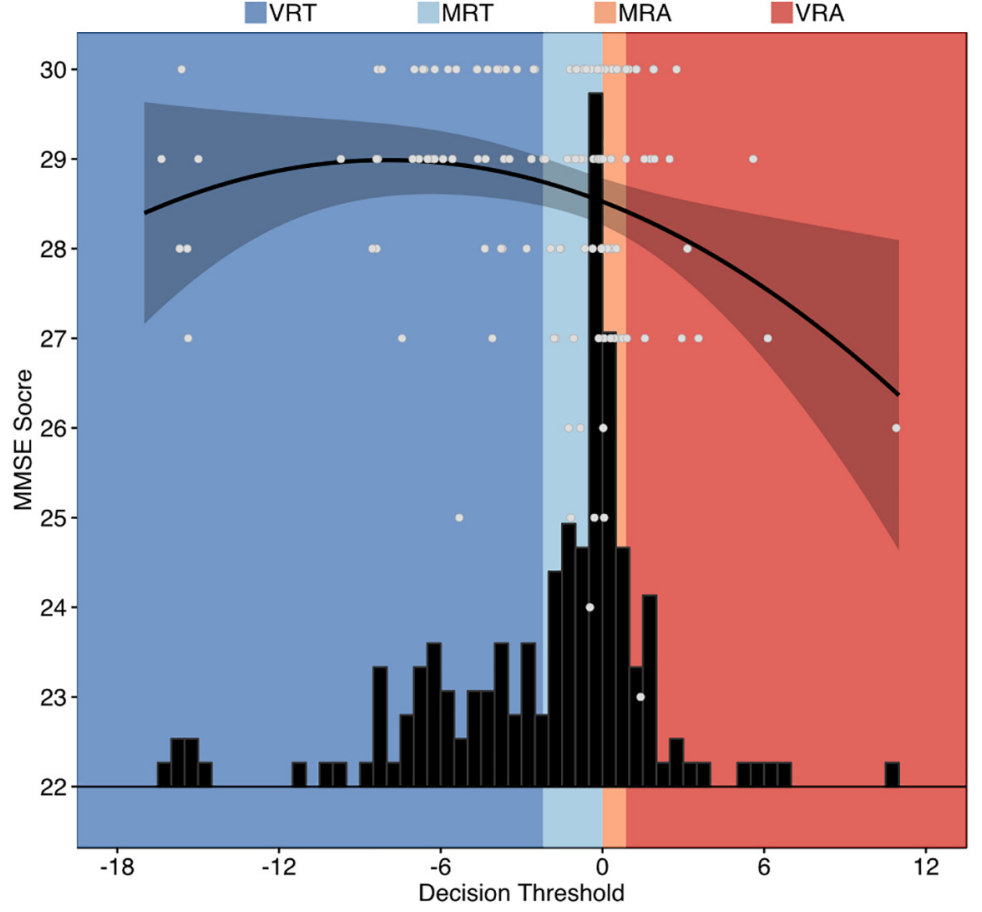

Figure 2. Histogram of DTs showing a substantial range of risk preferences in the sample. The modeled association between DT and MMSE performance is also shown with $95 \% \mathrm{Cl}$ along with individual data (gray dots). MMSE score was maximum for DT = -7.4 but lower with more extreme DTs. For visualization, data are overlaid over four increasing levels of DT from VRT, to MRT, to

mum when DT $=-7.4$ and was lower with more extreme risk preferences, particularly for risk-averters.

\section{Brain regions showing common EV sensitivity during choice evaluation}

A number of regions in the frontal, striatal, and medial temporal areas were sensitive to EV variation at the group level regardless of age and individual differences in risk preference (Fig. 4A; Table 3). Specifically, we found increasing blood-oxygen-leveldependent (BOLD) signal as trials became more desirable with increasing EVs (positive EV sensitivity) in the right inferior frontal gyrus (pars orbitalis), left caudate, and a region spanning the ventral anterior cingulate and medial prefrontal cortex, as expected. In addition, we observed increasing BOLD signal as trials became more costly with decreasing EV (negative EV sensitivity) in the bilateral putamen, thalamus, right middle frontal gyrus, left inferior frontal gyrus (pars opercularis), and hippocampus.

\section{Brain regions where age and individual differences in risk} preference modulated EV sensitivity during choice evaluation Greater age was associated with greater negative EV sensitivity (increasing BOLD signal as trials became more costly) in the bilateral middle frontal, left superior medial frontal, and middle cingulate regions (Fig. 4B; Table 4). Critically, there was a distinct set of regions within the frontal, striatal, and medial temporal areas in which individual differences in risk preference significantly dissociated neural sensitivity to EVs (Fig. 5; Table 5). These regions included the anterior cingulate region (overlapping but posterior to that observed for the group response to EV above), medial superior frontal gyrus, bilateral parahippocampal gyri (entorhinal region), right orbitofrontal gyrus and caudate, and left thalamus and putamen. In all these 
A

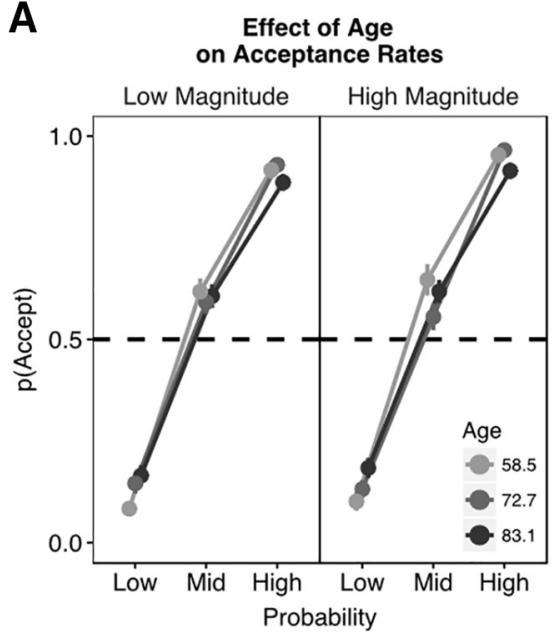

D

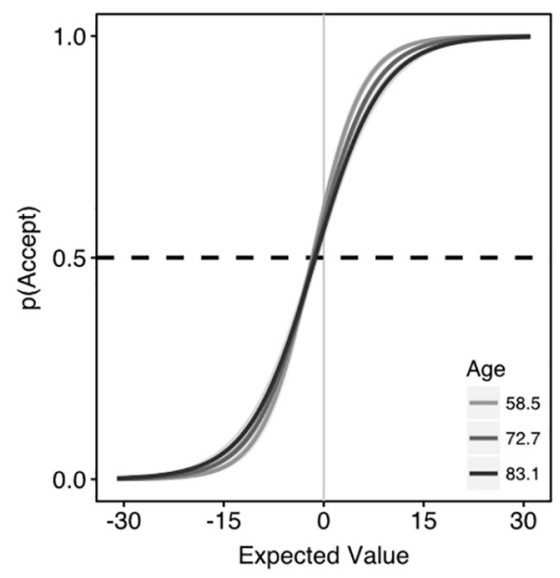

B

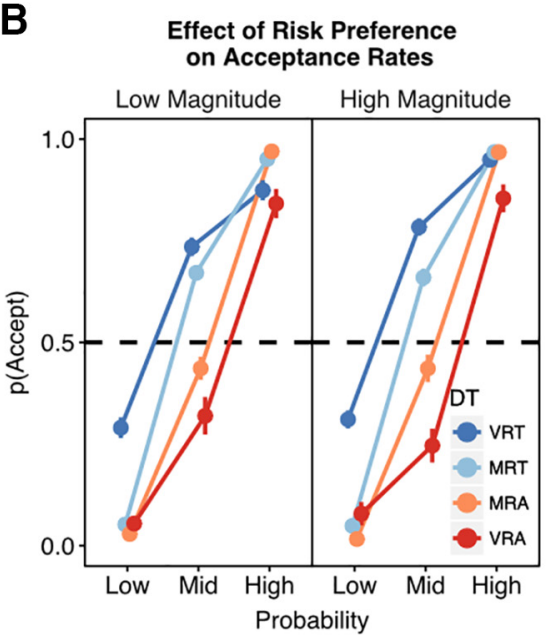

E

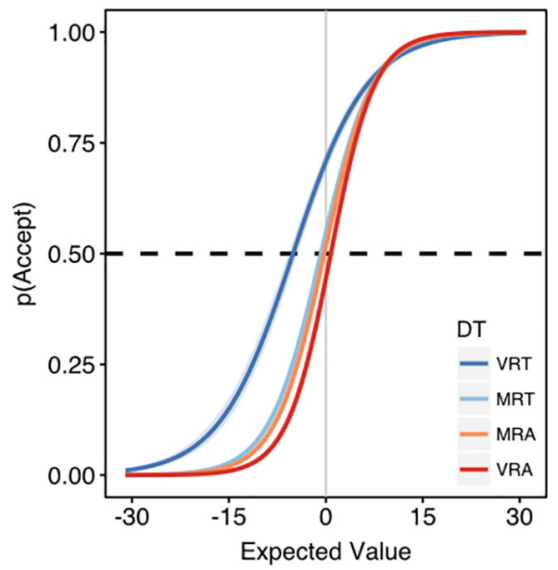

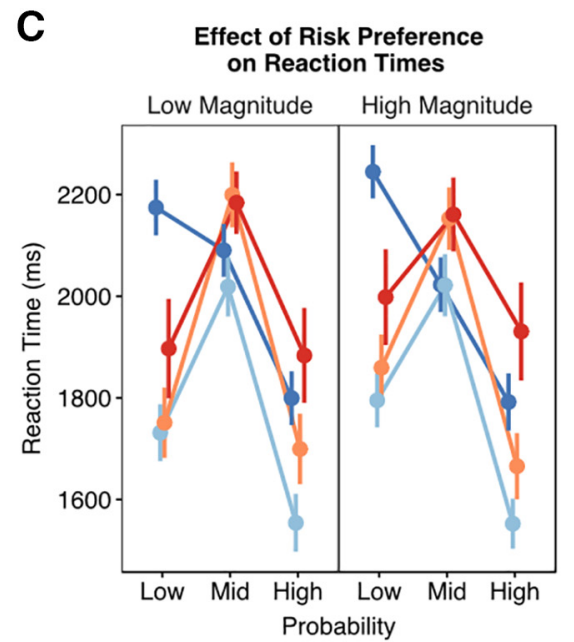

$\mathbf{F}$

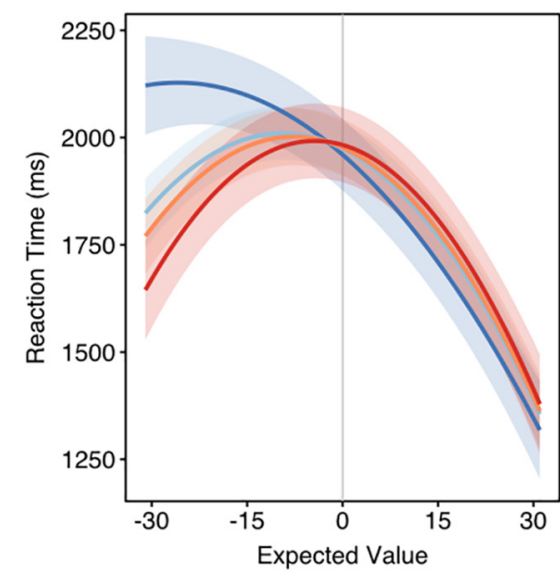

Figure 3. Behavioral performance across older individuals in the lottery choice task. $\boldsymbol{A}-\boldsymbol{C}$, Mean responses in each of the six choice conditions for $(\boldsymbol{A})$ acceptance rates over three incremental tertiles of age (legend shows mean age of each tertile), $(\boldsymbol{B})$ acceptance rates over the four DT levels, and ( $\boldsymbol{C}$ ) response times over the four DT levels. Error bars reflect $S E$. $\boldsymbol{D}-\boldsymbol{F}$, Modeled mean responses with $(\boldsymbol{D})$ acceptance rates over EV showing more suboptimal decision behavior with greater age, $(\boldsymbol{E})$ acceptance rates over EV showing increase in acceptance rates for costly trials as DT decreased, and $(\boldsymbol{F})$ response times over EV showing quadratic profiles with responses that became slower toward costly trials as DT decreased. Error bands reflect $95 \%$ Cls.

areas, greater risk-aversion was positively correlated with greater neural EV sensitivity with no regions showing negative associations.

Functional responses in ROIs defined from the above frontal, medial temporal, and striatal regions were plotted as a function of risk preferences to visualize the effect of DT in modulating neural activity across the range of EVs used in the lottery choice task in these brain regions (Fig. 5). For frontal ROIs, we found an overall pattern of positive EV sensitivity at the VRA, MRA, and MRT levels and negative EV sensitivity at the VRT level (VRA, MRA, MRT $>0$; VRT $<0$; Fig. 5A). Further, whereas the effects of risk preference in medial frontal ROIs were more prominent during positive EVs, effects of risk preference were more prominent during negative EVs in the right orbitofrontal ROI. In parahippocampal ROIs, the VRA and MRA levels showed positive EV sensitivity, with minimal bias at the MRT level, and negative EV sensitivity at the VRT level (VRA, MRA $>0 ; \mathrm{MRT} \approx 0$; VRT $<0$; Fig. 5B). Finally, in striatal ROIs, only the VRA level showed positive EV sensitivity, with minimal biases at the MRA and MRT levels, and negative sensitivity at the VRT level (VRA > 0; MRA, $\mathrm{MRT} \approx 0$; VRT $<0$; Fig. 5C).

Overall, these findings demonstrate that during choice evaluation, additional specific frontal, striatal, and medial temporal areas are involved in modulating neural responses across individuals over and above the common EV neural responses in these brain structures. Importantly, relative to their more risk-neutral counterparts, risk-aversive individuals showed accentuated sensitivity to EV in these additional brain areas, whereas risk-takers showed greater responses as decisions became more costly.

\section{Risk preferences modulate striatal prediction error during feedback}

Four ROIs fulfilled the conjunction criteria for canonical prediction error during feedback specified as positive responses to gains and negative responses to losses relative to fixation baseline. These included bilateral NAcc and the middle and posterior cingulate gyri. We found no significant effects of age on feedback responses in these four ROIs. Nevertheless, of these ROIs, risk preference significantly modulated feedback responses only in the bilateral NAcc (Fig. 6). Specifically, gain-loss contrasts were minimal for participants with DTs $\sim 0$, but increased toward more extreme DTs [(gain-loss $)^{2}$ : left, $t_{(168)}=3.47, p<0.001$; right, $\left.t_{(168)}=2.80, p<0.01\right]$. This effect of risk preference was driven mainly by increases in positive responses to gains in more risk-taking participants with lower DTs ( gain $^{2}$ : left, $t_{(168)}=2.30$, 


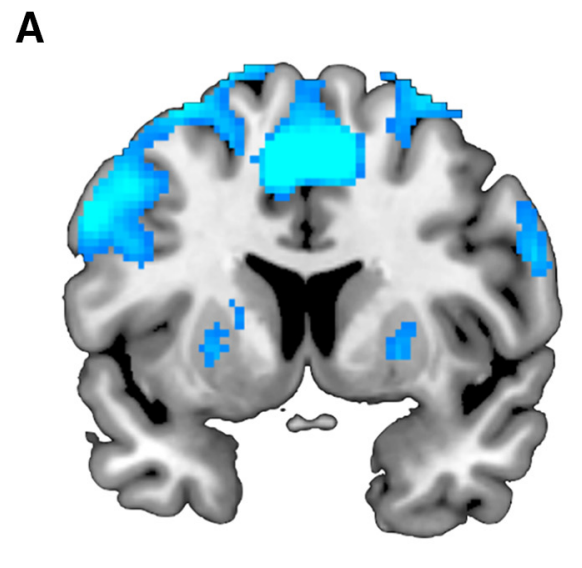

$y=8$

\section{B}

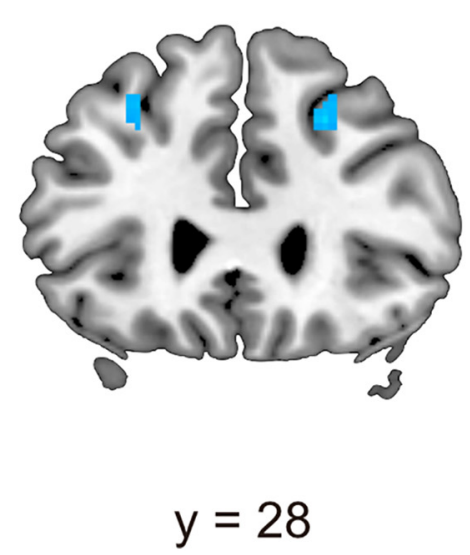

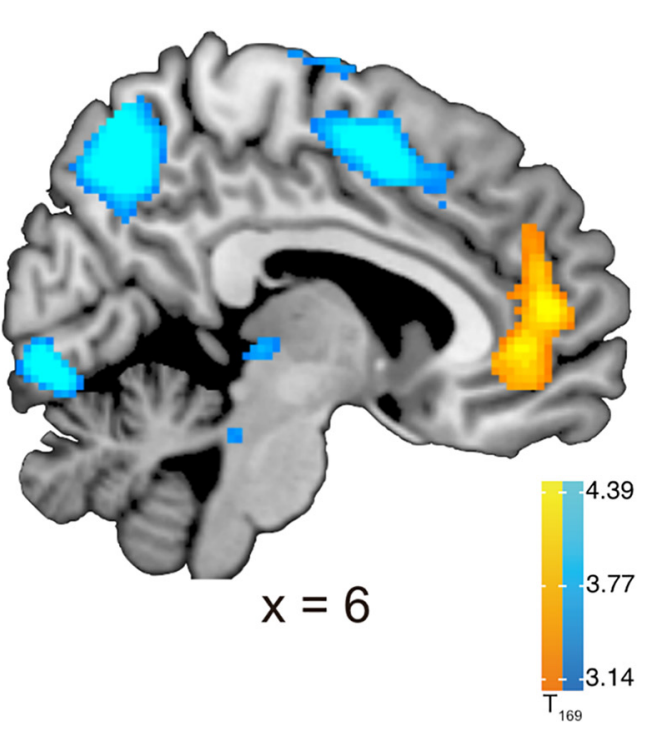

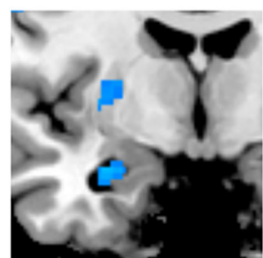

$y=-6$

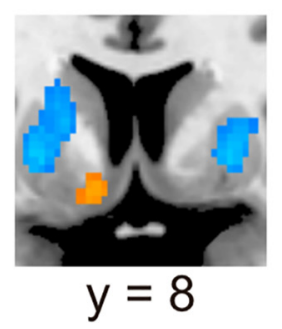

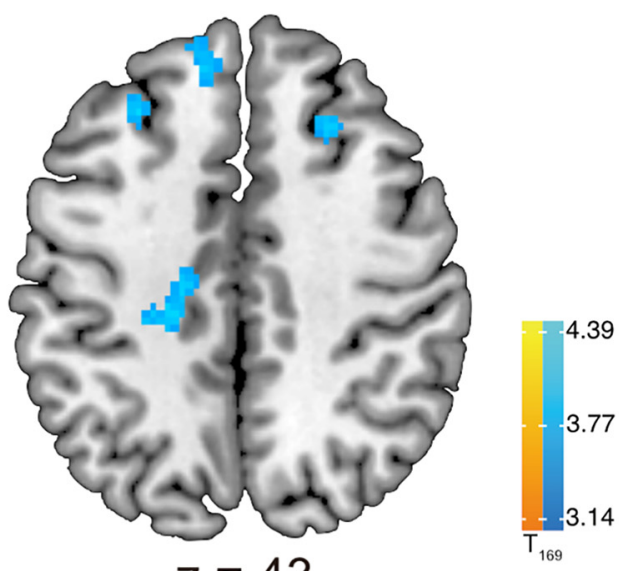

Figure 4. $\quad A, B$, Statistical map overlays on brain slices showing brain regions where $(\boldsymbol{A})$ individuals commonly engaged in the same manner with respect to the $\mathrm{EV}$ of stimuli, with increasing trial-by-trial EVs associated with increasing BOLD responses in warm-colored areas and decreasing BOLD responses in cool-colored areas, and (B) there were increasing BOLD responses to decreasing EVs that were accentuated with greater age. Significance threshold set at $p<0.001$; cluster size, $>20$ for cortical regions; $p<0.005$; cluster size, $>15$ for subcortical regions.

$p<0.05$; right, $\left.t_{(168)}=2.07, p<0.05\right)$ but minimal effects in negative responses to losses.

\section{Discussion}

Our results show that with greater age, value-based decisions become suboptimal with increased frontal responses to costlier choices. Given this, we examined individual differences in valuebased decision processing to better understand how risk preferences are associated with functional differences in frontal, striatal, and medial temporal areas. All participants were exposed to the same ranges of desirable to costly EVs, clearly delineated by explicit probabilities of winning (or losing) and magnitudes. Within this context, although participants increased acceptance rates as EVs increased, this sample of middle age and older participants evinced a preference for risk taking as a group. Crucially, while at least a half of the sample still accepted risks when losses were likely, at least a third demonstrated a reluctance to accept risks when gains were likely. This variability in risk preferences was associated with individual differences in frontal, striatal, and medial temporal responses to EV during choice evaluation distinct from general group-level EV-related responses. Specifically, neural activity in risk-averters increased with increasingly desirable EVs, with accentuated sensitivity compared with their neutral counterparts. By contrast, risk-takers increased neural activity with increasingly costly EVs, consistent with a propensity for experiencing gains under risk. Finally, risk preferences modulated prediction error responses during feedback, with risktakers showing more striatal activation to gains, possibly signaling greater surprise.

Our results are consistent with an increase in neural activity to increasing stimulus value in the frontostriatal areas (Schultz et al., 1997; Breiter et al., 2001; Knutson et al., 2001; Fiorillo et al., 2003; Preuschoff et al., 2006; Tobler et al., 2007; Tom et al., 2007). Such correspondence between neural activity and assigned stimulus values in these regions during choice evaluation reflects a neural mechanism for the comparative representation of anticipated rewards or costs, particularly for the likelihood of occurrence of the reward or cost (Fiorillo et al., 2003). Our findings extend this notion by showing that there are also important individual differences in the correspondence between neural activity in these regions and stimulus values that are associated with differences in 
Table 3. Peak voxels in which EV modulated BOLD responses after accounting for DT and age with sex as a covariate

\begin{tabular}{|c|c|c|c|c|c|c|c|c|c|}
\hline \multirow[b]{2}{*}{ Region } & \multirow[b]{2}{*}{ Hemisphere } & \multirow[b]{2}{*}{$x$} & \multirow[b]{2}{*}{$y$} & \multirow[b]{2}{*}{$z$} & \multirow[b]{2}{*}{$\mathrm{BA}$} & \multirow[b]{2}{*}{ Cluster size } & \multirow[b]{2}{*}{$t$ value } & \multicolumn{2}{|l|}{$P$} \\
\hline & & & & & & & & Uncorrected & False discovery rate \\
\hline Anterior cingulate gyrus & Left, right & 2 & 44 & 0 & $32,11,10$ & 1168 & 5.29 & $<0.001$ & $<0.01$ \\
\hline Lingual gyrus & Left & -10 & -82 & -10 & 18 & 490 & 7.76 & $<0.001$ & $<0.001$ \\
\hline Caudate & Left & -12 & 8 & -10 & - & 27 & 3.15 & $<0.001$ & 0.651 \\
\hline Inferior frontal gyrus (pars orbitalis) & Right & 28 & 30 & -14 & 47 & 96 & 4.65 & $<0.001$ & 0.049 \\
\hline Middle temporal gyrus & Right & 54 & -18 & -8 & 22 & 44 & 4.07 & $<0.001$ & 0.283 \\
\hline Calcarine fissure & Right & 30 & -54 & 10 & 19 & 23 & 3.52 & $<0.001$ & 0.703 \\
\hline Supplementary motor area & Left, right & -6 & 12 & 50 & $32,24,6$ & 14,368 & -6.53 & $<0.001$ & $<0.001$ \\
\hline Precuneus & Left, right & 6 & -60 & 50 & 7 & & -6.28 & $<0.001$ & $<0.001$ \\
\hline Inferior frontal gyrus (pars opercularis) & Left & -48 & 14 & 20 & 44 & & -4.56 & $<0.001$ & 0.189 \\
\hline Precentral gyrus & Left & -58 & 2 & 34 & 4 & & -5.26 & $<0.001$ & 0.023 \\
\hline Postcentral gyrus & Left & -38 & -32 & 44 & $40,3,2$ & & -6.35 & $<0.001$ & $<0.001$ \\
\hline Middle occipital gyrus & Right & 32 & -72 & 18 & 19 & & -5.75 & $<0.001$ & 0.006 \\
\hline Postcentral gyrus & Right & 40 & -28 & 46 & 3,2 & & -5.63 & $<0.001$ & 0.003 \\
\hline Superior frontal gyrus & Right & 30 & 0 & 68 & 6 & & -5.49 & $<0.001$ & 0.004 \\
\hline Thalamus & Left, right & -12 & -20 & 10 & - & 1013 & -4.87 & $<0.001$ & 0.010 \\
\hline Fusiform gyrus & Left & -38 & -56 & -12 & 37 & 193 & -4.27 & $<0.001$ & 0.116 \\
\hline Middle temporal gyrus & Left & -46 & -40 & -2 & 21 & 123 & -4.65 & $<0.001$ & 0.044 \\
\hline Hippocampus & Left & -30 & -6 & -20 & 20 & 27 & -3.42 & $<0.001$ & 0.265 \\
\hline Lingual gyrus & Left & -22 & -60 & 0 & 19 & 37 & -3.79 & $<0.001$ & 0.308 \\
\hline Putamen & Left & -20 & 4 & 6 & - & 639 & -3.82 & $<0.001$ & 0.132 \\
\hline Putamen & Right & 24 & 6 & 0 & - & 242 & -3.69 & $<0.001$ & 0.163 \\
\hline Precentral gyrus & Right & 60 & 6 & 24 & 44,6 & 227 & -4.33 & $<0.001$ & 0.104 \\
\hline Middle frontal gyrus & Right & 46 & 32 & 36 & 45 & 22 & -3.56 & $<0.001$ & 0.438 \\
\hline Lingual gyrus & Right & 12 & -82 & -6 & 18 & 1778 & -10.47 & $<0.001$ & $<0.001$ \\
\hline
\end{tabular}

Table 4. Peak voxels in which age modulated BOLD responses after accounting for group EV responses and DT with sex as a covariate

\begin{tabular}{|c|c|c|c|c|c|c|c|c|c|}
\hline \multirow[b]{2}{*}{ Region } & \multirow[b]{2}{*}{ Hemisphere } & \multirow[b]{2}{*}{$x$} & \multirow[b]{2}{*}{$y$} & \multirow[b]{2}{*}{$z$} & \multirow[b]{2}{*}{ BA } & \multirow[b]{2}{*}{ Cluster size } & \multirow[b]{2}{*}{$t$ value } & \multicolumn{2}{|l|}{$P$} \\
\hline & & & & & & & & Uncorrected & False discovery rate \\
\hline Middle frontal gyrus & Right & 24 & 26 & 40 & 9 & 43 & -4.05 & $<0.001$ & 0.437 \\
\hline Middle frontal gyrus & Left & -28 & 30 & 42 & 9 & 68 & -3.85 & $<0.001$ & 0.437 \\
\hline Superior medial frontal gyrus & Left & -10 & 42 & 42 & 9 & 50 & -3.70 & $<0.001$ & 0.491 \\
\hline Middle cingulate gyrus & Left & -16 & -16 & 44 & 23 & 96 & -4.02 & $<0.001$ & 0.437 \\
\hline
\end{tabular}

relative value comparisons. We also show that increasing potential cost induced increasing neural activity (negative EV sensitivity) in the putamen, thalamus, lateral frontal, and hippocampal areas. This finding contrasts with a previous study that did not detect any negative correlations between brain responses and increasingly negative stakes (Tom et al., 2007), although this may be due to asymmetry in the range of gains and losses, a relatively small sample size, and the lack of outcomes in that study (Canessa et al., 2013; Sokol-Hessner et al., 2013). Our observations of negative EV sensitivity in these regions are consistent with their involvement in emotional regulation, which reduces loss aversion and may therefore increase risk-taking behavior (Knoch et al., 2006; Baumgartner et al., 2011; Sokol-Hessner et al., 2013). Note, risk preference effects on behavior were more pronounced during negative EVs, which might be affected by individual differences in loss aversion. Moreover, increased dorsolateral prefrontal activity is observed in individuals who have a propensity for more costly decisions (Baumgartner et al., 2011). Together, these findings may reflect the engagement of neural resources in some individuals to regulate experienced inconsistency between subjective goals and the options at hand. Our study and other supportive findings (Tobler et al., 2009) buttress this notion in that risk-takers but not risk-averters displayed increasing frontal, striatal, and hippocampal neural activity as decisions were associated with greater costs. Future studies directly evaluating loss aversion or other aspects of decision processing are required to determine the submechanisms operating in these regions that underlie the observed differences.
That older neural decision-processing differences were present during the choice phase suggests individually different value perceptions of stimuli during predictive processing. This is distinct from the effect of declines in feedback learning on the decisions of older adults (Denburg et al., 2005; Mata et al., 2011), as is typically tested with stochastic learning paradigms, such as the Iowa Gambling Task. Indeed, there were no age effects on neural responses during feedback in our task. As demonstrated in a study in younger adults with different decision strategies (Venkatraman et al., 2009), distortion of value information during the choice stage may carry into feedback so that prediction error is different (but not necessarily distorted) across older individuals with different risk preferences. Thus, our findings are consistent with individual differences in age-related effects on frontal, striatal, and medial temporal processing that influence older-adult value processing and affect feedback processing but are distinct from age effects on feedback learning.

Our findings point to a possible functional neural mechanism underlying individual differences in older adults displaying variability in risk-taking behaviors (Denburg et al., 2005) and, possibly, responses to drugs (Chowdhury et al., 2013) that affect neurotransmitter action, such as those that affect the action of dopamine (Wang et al., 1995; Volkow et al., 1998; Kaasinen et al., 2000; Erixon-Lindroth et al., 2005; Dreher et al., 2008). Dopamine efficacy is optimal at moderate levels of synaptic availability but dysregulated at both lower and higher levels (Goldman-Rakic et al., 2000). We speculate that the distribution of neural sensitivity to value in our study reflects a functional correlate of dif- 
A

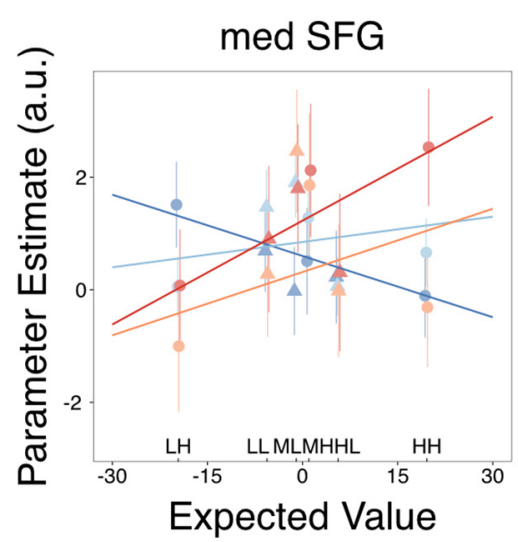

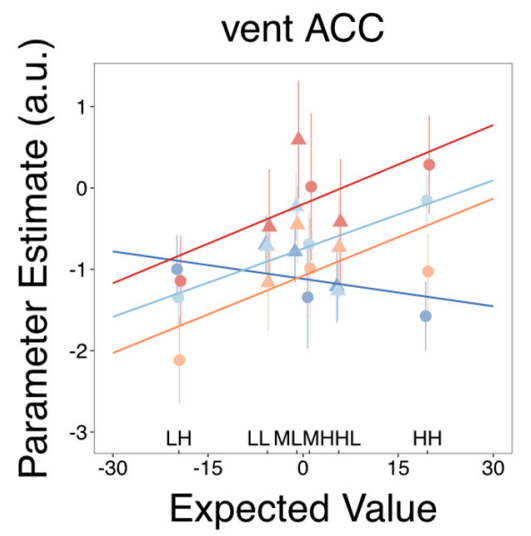
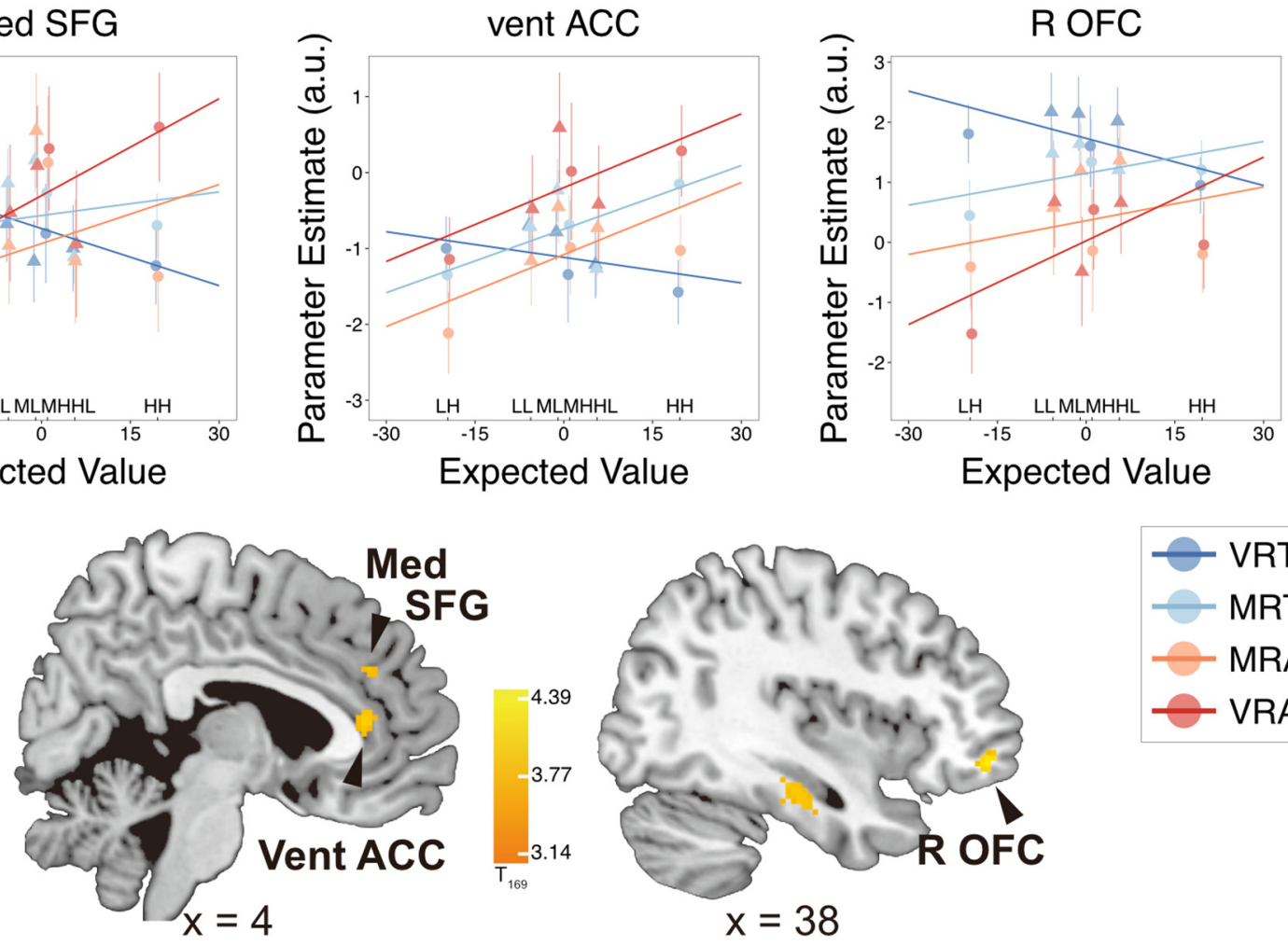

V VRT

- MRT

- MRA

- VRA

B

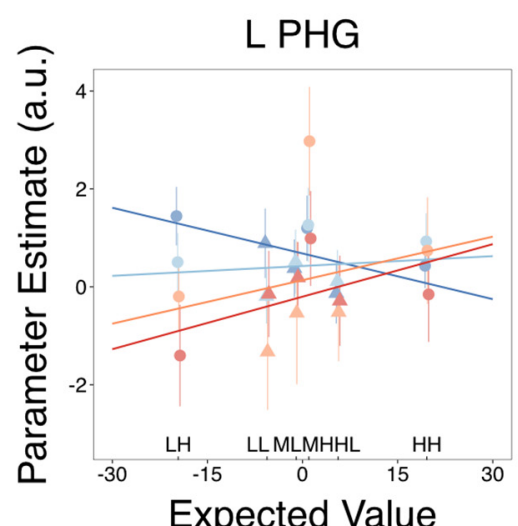

C

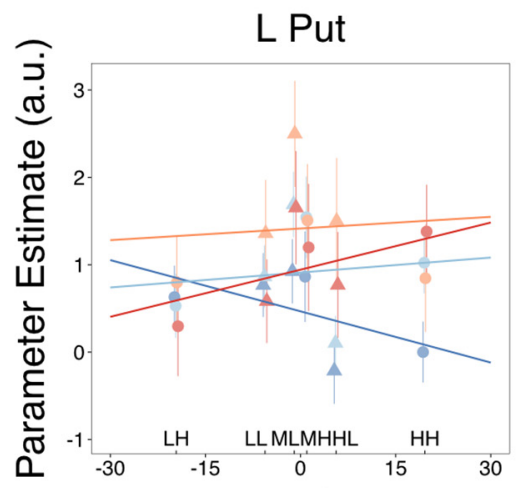

Expected Value
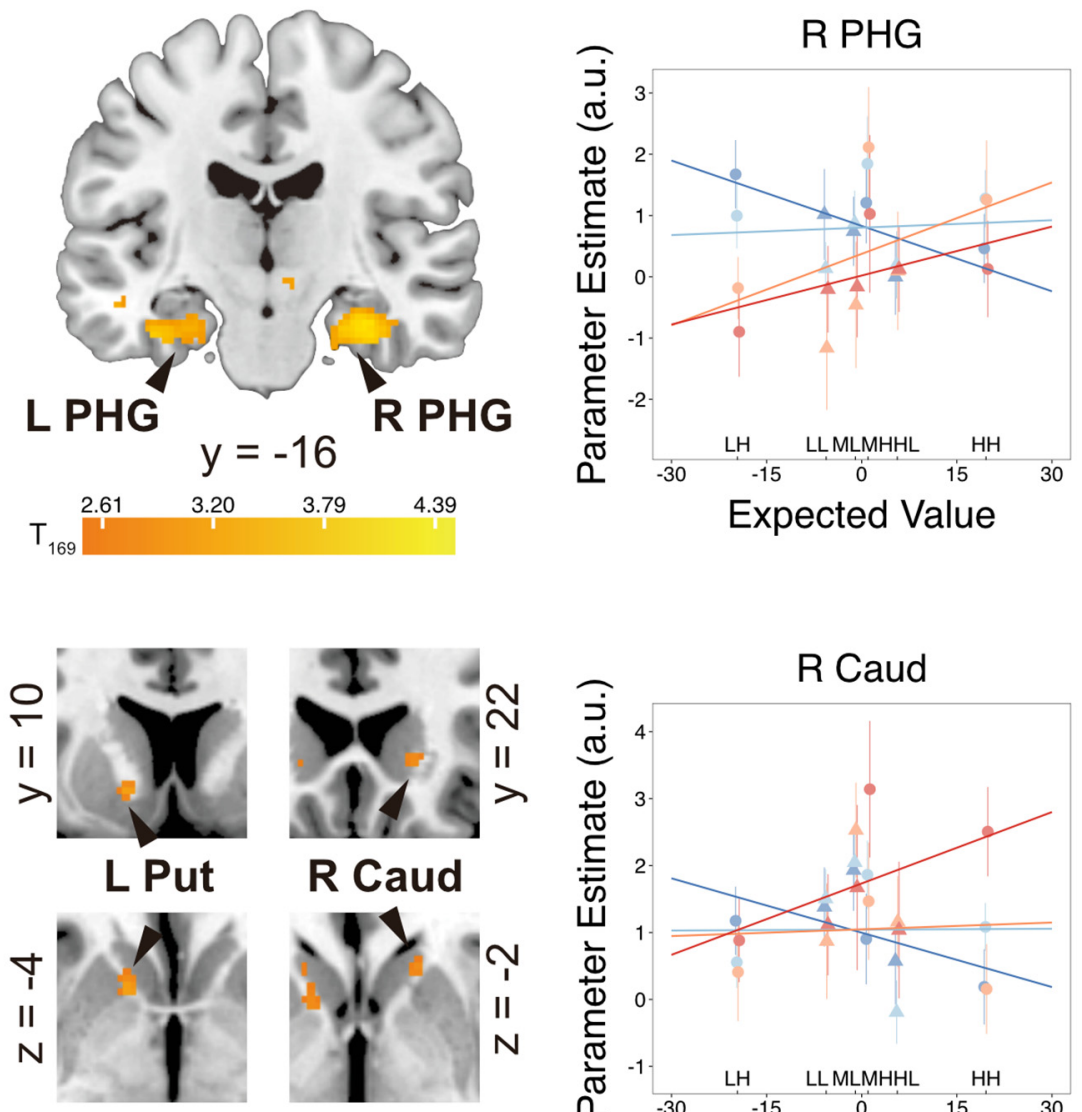

$T_{169}^{2.61}$

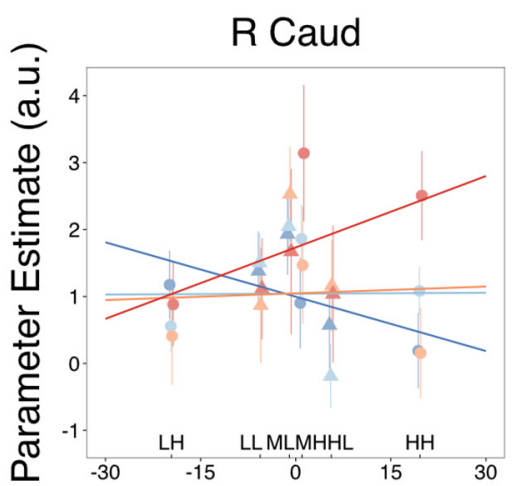

Expected Value 
Table 5. Peak voxels in which DT modulated neural sensitivity to EV, adjusted for mean neural responses to EV, age, and sex

\begin{tabular}{|c|c|c|c|c|c|c|c|c|c|}
\hline \multirow[b]{2}{*}{ Regions } & \multirow[b]{2}{*}{ Hemisphere } & \multirow[b]{2}{*}{$x$} & \multirow[b]{2}{*}{$y$} & \multirow[b]{2}{*}{$z$} & \multirow[b]{2}{*}{ BA } & \multirow[b]{2}{*}{ Cluster size } & \multirow[b]{2}{*}{$t$ value } & \multicolumn{2}{|l|}{$P$} \\
\hline & & & & & & & & Uncorrected & False discovery rate \\
\hline Medial superior frontal cingulate gyrus ${ }^{a}$ & Left, right & 0 & 38 & 30 & 32 & 28 & 3.70 & $<0.001$ & 0.922 \\
\hline Anterior cingulate gyrus ${ }^{a}$ & Left, right & -6 & 38 & 14 & 32,24 & 121 & 4.19 & $<0.001$ & 0.702 \\
\hline Parahippocampal gyrus ${ }^{a}$ & Left & -38 & -20 & -24 & $36,35,20$ & 73 & 3.86 & $<0.001$ & 0.922 \\
\hline Putamen $^{a}$ & Left & -14 & 10 & -4 & - & 26 & 3.12 & $<0.001$ & 0.819 \\
\hline Thalamus & Left & -14 & -10 & 4 & - & 18 & 3.13 & $<0.001$ & 0.819 \\
\hline Orbitofrontal gyrus $^{a}$ & Right & 38 & 50 & -10 & 47 & 46 & 4.34 & $<0.001$ & 0.702 \\
\hline Parahippocampal gyrus ${ }^{a}$ & $\mathrm{R}$ & 32 & -16 & -22 & $36,35,20$ & 250 & 4.06 & $<0.001$ & 0.702 \\
\hline Inferior temporal gyrus & $\mathrm{R}$ & 44 & -6 & -44 & 20 & 21 & 4.15 & $<0.001$ & 0.702 \\
\hline Caudate $^{a}$ & $\mathrm{R}$ & 18 & 22 & -2 & - & 15 & 2.86 & $<0.002$ & 0.819 \\
\hline
\end{tabular}

a Peak coordinates used to define ROls in these regions.

A

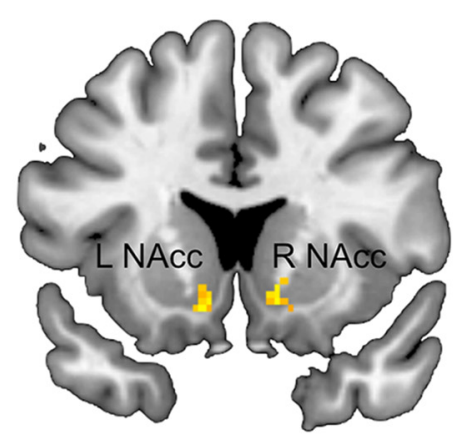

B

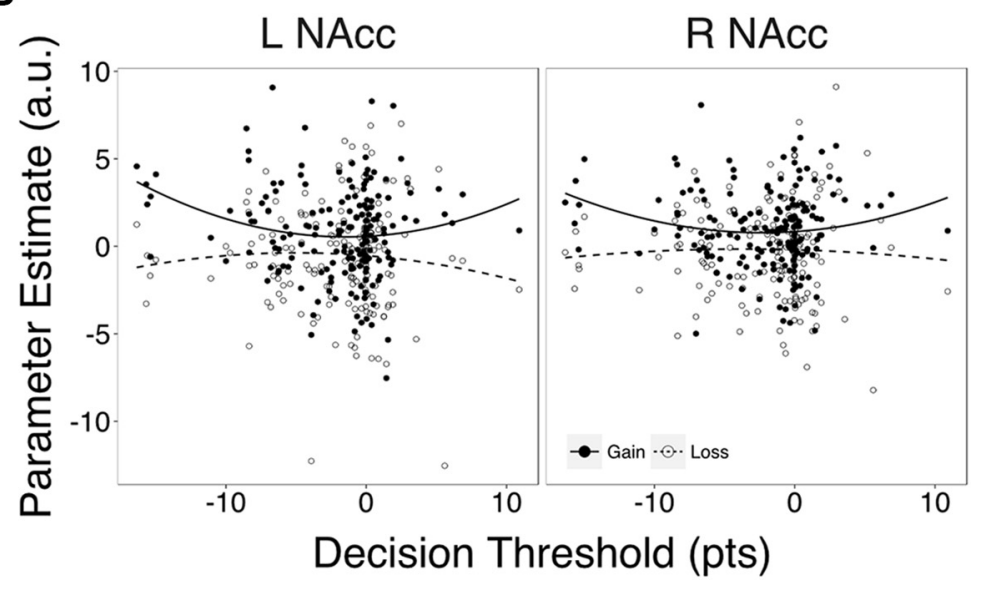

Figure 6. Feedback-related responses in ROIs identified from a whole-brain conjunction analysis of enhanced and reduced responses to gain and loss, respectively, relative to fixation baseline. $\boldsymbol{A}$, DT significantly modulated feedback-related responses only in the left and right NAcc in mixed-model analyses. $\boldsymbol{B}$, Measured individual and modeled feedback responses to gain and loss are plotted for each ROI. Feedback responses were minimal in participants with DTs close to zero, but showed quadratic increase toward extreme risk preferences. In particular, risk-seeking participants with low DT showed significantly accentuated enhancement of responses to gains. Significance threshold set at $p<0.001$, cluster size, $>20$.

ferences in dopamine levels at the lower end of the availability spectrum among older individuals. Specifically, older adults with moderate neural value sensitivity and decision behaviors in line with objective stimuli values may have optimal dopaminergic efficacy. By contrast, older adults with disproportionate neural value sensitivity or who show more responsiveness to costs than rewards may have dysregulated dopamine efficacy associated with noisier signaling (Eppinger et al., 2011). Only the latter, with irregular value sensitivity, should show more accurate value representations when dopamine antagonists or agonists are introduced that improve signal gain accordingly. Indeed, higher frontostriatal dopamine levels correlate with greater willingness to expend effort for gains (more risk-taking; Treadway et al., 2012) and higher frontal activity in young adults (Dreher et al.,

\footnotetext{
Figure 5. $\quad \boldsymbol{A}-\boldsymbol{C}$, Statistical overlays on brain slices showing $(\boldsymbol{A})$ frontal, $(\boldsymbol{B})$ limbic, and $(\boldsymbol{C})$ ventral striatal ROls where individual differences in risk preferences (DT) influenced neural sensitivity to EV. Modeled brain responses over trial-wise EVs are plotted over four levels of risk preferences for each ROI. Mean brain response estimates (error bars denote SE) for the six discrete choice conditions (HH, MH, LH as circles; HL, ML, LL as triangles; Table 2) are also overlaid to aid comparison between modeled and measured results. Note that choice condition estimates are plotted against approximately representative EVs on the abscissa and are not evenly distributed. SFG, Superior frontal gyrus; $\mathrm{ACC}$, anterior cingulate cortex; $\mathrm{OFC}$, orbitofrontal cortex; PHG, parahippocampal gyrus; Put, putamen; Caud, caudate. Significance threshold set at $p<0.001$; cluster size, $>20$ for cortical regions; $p<0.005$, cluster size $>15$ for subcortical regions.
}

2008). However, in older adults, higher midbrain dopaminergic synthesis correlates with lower frontal activity (Dreher et al., 2008), consistent with having lower baseline dopamine levels. Further studies involving direct manipulation of dopamine levels in older adults are necessary to validate this hypothesis.

Many regions in which risk preferences modulated neural value processing in our study have been implicated as initiating loci for neuropathological progressions in various cognitive diseases. For example, medial temporal neurofibrillary tangles and medial frontal and striatal $\beta$-amyloid deposition occur in early stages of Alzheimer's disease (Braak and Braak, 1995, 1997). The loss of dopaminergic receptors (Wong et al., 1984; Wang et al., 1995; Volkow et al., 1998; Kaasinen et al., 2000; Erixon-Lindroth et al., 2005; Dreher et al., 2008) along with iron accumulation (which increases oxidative stress; Bartzokis et al., 1997; Pfefferbaum et al., 2010) in the striatal regions are also among the most reliably observed neurobiological changes associated with aging. Interestingly, in our study on cognitively normal older adults, individuals with more extreme risk preferences evinced poorer MMSE performance, with a tendency for those with a slight preference for risk to have the highest scores. Overall, the dissociated frontal, striatal, and medial temporal functional responses between risk-takers and risk-averters with opposite sensitivities to $\mathrm{EV}$ in our study may form a basis for a preclinical biomarker of age-related cognitive impairment. 
Given the mixed findings on age effects on risk preferences that might stem from differences in decision contexts (Mata et al., 2011), key aspects of our design should be highlighted. First, we used numerical texts to explicitly depict value magnitudes and probabilities in each trial. Second, all information needed to make a decision was available in the stimuli, which were independent across trials, to minimize any involvement of learning and memory. Using any learning of past decision outcomes would be fallacious and a suboptimal strategy in the task. Third, we used mixed stakes that simultaneously included the possibility for wins and losses in every decision fairly across trials. Our design contrasts with studies that involved proxy representations of value, learning, or incorporation of outcome history, or separate losing and winning trials, or only wins and forfeitures. This last point in particular, was important for assessing individual risk preferences in older adults during costly but potentially tempting choices (mixed stakes), which have been associated with increased risk-taking with age (Rutledge et al., 2016). We note that our data are based on the BLSA, which consists of participants who choose to enroll in intensive physical and behavioral assessments and may reflect personalities associated with a risk-taking preference. Ongoing studies are investigating the extent to which these individual differences in frontal, striatal, and medial temporal functional activity are generalizable to other samples.

How a person makes decisions about value reveals much about the ability of his or her brain to regulate and integrate subjective goals with objective information. While we found that age generally compromises value-based decision behavior and neural processing, our study further links individual differences in older-adult risk preferences to differences in frontal, striatal, and medial temporal responses to value information during decision processing. These risk preference-related neural responses in older adults range from hypersensitivity to value in riskaverters to greater sensitivity to costly choices instead in risktakers. Such patterns of neural responses are obscured in analyses focusing on common patterns of responses in groups of individuals, highlighting the importance of investigating individual differences to understand the neurobiology of decision making in older adults. Altered value representation in this neural system may indicate susceptibility to age-related changes in some older individuals resulting in biased risk preferences that compromise decision-making skills critical for optimal lifestyles in stochastic environments.

\section{References}

Ashburner J (2007) A fast diffeomorphic image registration algorithm. Neuroimage 38:95-113. CrossRef Medline

Bartzokis G, Beckson M, Hance DB, Marx P, Foster JA, Marder SR (1997) MR evaluation of age-related increase of brain iron in young adult and older normal males. Magn Reson Imaging 15:29-35. CrossRef Medline

Baumgartner T, Knoch D, Hotz P, Eisenegger C, Fehr E (2011) Dorsolateral and ventromedial prefrontal cortex orchestrate normative choice. Nat Neurosci 14:1468-1474. CrossRef Medline

Beason-Held LL, Goh JO, An Y, Kraut MA, O’Brien RJ, Ferrucci L, Resnick SM (2013) Changes in brain function occur years before the onset of cognitive impairment. J Neurosci 33:18008-18014. CrossRef Medline

Braak H, Braak E (1995) Staging of Alzheimer's disease-related neurofibrillary changes. Neurobiol Aging 16:271-284. CrossRef Medline

Braak H, Braak E (1997) Frequency of stages of Alzheimer-related lesions in different age categories. Neurobiol Aging 18:351-357. CrossRef Medline

Breiter HC, Aharon I, Kahneman D, Dale A, Shizgal P (2001) Functional imaging of neural responses to expectancy and experience of monetary gains and losses. Neuron 30:619-639. CrossRef Medline

Canessa N, Crespi C, Motterlini M, Baud-Bovy G, Chierchia G, Pantaleo G, Tettamanti M, Cappa SF (2013) The functional and structural neural basis of individual differences in loss aversion. J Neurosci 33:1430714317. CrossRef Medline

Chowdhury R, Guitart-Masip M, Lambert C, Dayan P, Huys Q, Düzel E, Dolan RJ (2013) Dopamine restores reward prediction errors in old age. Nat Neurosci 16:648-653. CrossRef Medline

Denburg NL, Tranel D, Bechara A (2005) The ability to decide advantageously declines prematurely in some normal older persons. Neuropsychologia 43:1099-1106. CrossRef Medline

Dohmen T, Falk A, Huffman D, Sunde U, Schupp J, Wagner GG (2011) Individual risk attitudes: measurement, determinants, and behavioral consequences. J Eur Econ Assoc 9:522-550. CrossRef

Dreher JC, Meyer-Lindenberg A, Kohn P, Berman KF (2008) Age-related changes in midbrain dopaminergic regulation of the human reward system. Proc Natl Acad Sci U S A 105:15106-15111. CrossRef Medline

Driscoll I, Resnick SM, Troncoso JC, An Y, O’Brien R, Zonderman AB (2006) Impact of Alzheimer's pathology on cognitive trajectories in nondemented elderly. Ann Neurol 60:688-695. CrossRef Medline

Eppinger B, Hämmerer D, Li SC (2011) Neuromodulation of reward-based learning and decision making in human aging. Ann N Y Acad Sci 1235: 1-17. CrossRef Medline

Erixon-Lindroth N, Farde L, Wahlin TB, Sovago J, Halldin C, Bäckman L (2005) The role of the striatal dopamine transporter in cognitive aging. Psychiatry Res 138:1-12. CrossRef Medline

Fiorillo CD, Tobler PN, Schultz W (2003) Discrete coding of reward probability and uncertainty by dopamine neurons. Science 299:1898-1902. CrossRef Medline

Goh JO (2011) Functional dedifferentiation and altered connectivity in older adults: neural accounts of cognitive aging. Aging Dis 2:30-48. Medline

Goh JO, Park DC (2009) Neuroplasticity and cognitive aging: the scaffolding theory of aging and cognition. Restor Neurol Neurosci 27:391-403. CrossRef Medline

Goh JO, An Y, Resnick SM (2012) Differential trajectories of age-related changes in components of executive and memory processes. Psychol Aging 27:707-719. CrossRef Medline

Goh JO, Beason-Held LL, An Y, Kraut MA, Resnick SM (2013) Frontal function and executive processing in older adults: process and region specific age-related longitudinal functional changes. Neuroimage 69:4350. CrossRef Medline

Goldman-Rakic PS, Muly EC 3rd, Williams GV (2000) D(1) receptors in prefrontal cells and circuits. Brain Res Rev 31:295-301. CrossRef Medline

Kaasinen V, Vilkman H, Hietala J, Någren K, Helenius H, Olsson H, Farde L, Rinne J (2000) Age-related dopamine D2/D3 receptor loss in extrastriatal regions of the human brain. Neurobiol Aging 21:683-688. CrossRef Medline

Kawas C, Gray S, Brookmeyer R, Fozard J, Zonderman A (2000) Agespecific incidence rates of Alzheimer's disease: The Baltimore Longitudinal Study of Aging. Neurology 54:2072-2077. CrossRef Medline

Knoch D, Gianotti LR, Pascual-Leone A, Treyer V, Regard M, Hohmann M, Brugger P (2006) Disruption of right prefrontal cortex by lowfrequency repetitive transcranial magnetic stimulation induces risktaking behavior. J Neurosci 26:6469-6472. CrossRef Medline

Knutson B, Adams CM, Fong GW, Hommer D (2001) Anticipation of increasing monetary reward selectively recruits nucleus accumbens. J Neurosci 21:RC159. Medline

Koster R, Guitart-Masip M, Dolan RJ, Düzel E (2015) Basal ganglia activity mirrors a benefit of action and reward on long-lasting event memory. Cereb Cortex 25:4908-4917. CrossRef Medline

Lieberman MD, Cunningham WA (2009) Type I and Type II error concerns in fMRI research: re-balancing the scale. Soc Cogn Affect Neurosci 4:423428. CrossRef Medline

Mata R, Josef AK, Samanez-Larkin GR, Hertwig R (2011) Age differences in risky choice: a meta-analysis. Ann N Y Acad Sci 1235:18-29. CrossRef Medline

Pfefferbaum A, Adalsteinsson E, Rohlfing T, Sullivan EV (2010) Diffusion tensor imaging of deep gray matter brain structures: effects of age and iron concentration. Neurobiol Aging 31:482-493. CrossRef Medline

Preuschoff K, Bossaerts P, Quartz SR (2006) Neural differentiation of expected reward and risk in human subcortical structures. Neuron 51:381390. CrossRef Medline

Resnick SM, Goldszal AF, Davatzikos C, Golski S, Kraut MA, Metter EJ, Bryan 
RN, Zonderman AB (2000) One-year age changes in MRI brain volumes in older adults. Cereb Cortex 10:464-472. CrossRef Medline

Rutledge RB, Smittenaar P, Zeidman P, Brown HR, Adams RA, Lindenberger U, Dayan P, Dolan RJ (2016) Risk taking for potential reward decreases across the lifespan. Curr Biol 26:1634-1639. CrossRef Medline

Samanez-Larkin GR, Gibbs SE, Khanna K, Nielsen L, Carstensen LL, Knutson B (2007) Anticipation of monetary gain but not loss in healthy older adults. Nat Neurosci 10:787-791. CrossRef Medline

Samanez-Larkin GR, Kuhnen CM, Yoo DJ, Knutson B (2010) Variability in nucleus accumbens activity mediates age-related suboptimal financial risk taking. J Neurosci 30:1426-1434. CrossRef Medline

Schultz W, Dayan P, Montague PR (1997) A neural substrate of prediction and reward. Science 275:1593-1599. CrossRef Medline

Slotnick SD (2011) Cluster_threshold. Available at: http://www2.bc.edu/ $\sim$ slotnics/scripts.htm. Accessed August 4, 2011.

Slotnick SD, Moo LR, Segal JB, Hart J Jr (2003) Distinct prefrontal cortex activity associated with item memory and source memory for visual shapes. Brain Res Cogn Brain Res 17:75-82. CrossRef Medline

Sokol-Hessner P, Camerer CF, Phelps EA (2013) Emotion regulation reduces loss aversion and decreases amygdala responses to losses. Soc Cogn Affect Neurosci 8:341-350. CrossRef Medline

Tobler PN, O’Doherty JP, Dolan RJ, Schultz W (2007) Reward value coding distinct from risk attitude-related uncertainty coding in human reward systems. J Neurophysiol 97:1621-1632. Medline

Tobler PN, Christopoulos GI, O’Doherty JP, Dolan RJ, Schultz W (2009) Risk-dependent reward value signal in human prefrontal cortex. Proc Natl Acad Sci U S A 106:7185-7190. CrossRef Medline
Tom SM, Fox CR, Trepel C, Poldrack RA (2007) The neural basis of loss aversion in decision-making under risk. Science 315:515-518. CrossRef Medline

Treadway MT, Buckholtz JW, Cowan RL, Woodward ND, Li R, Ansari MS, Baldwin RM, Schwartzman AN, Kessler RM, Zald DH (2012) Dopaminergic mechanisms of individual differences in human effort-based decision-making. J Neurosci 32:6170-6176. CrossRef Medline

Tzourio-Mazoyer N, Landeau B, Papathanassiou D, Crivello F, Etard O, Delcroix N, Mazoyer B, Joliot M (2002) Automated anatomical labeling of activations in SPM using a macroscopic anatomical parcellation of the MNI MRI single-subject brain. Neuroimage 15:273-289. CrossRef Medline

Venkatraman V, Payne JW, Bettman JR, Luce MF, Huettel SA (2009) Separate neural mechanisms underlie choices and strategic preferences in risky decision making. Neuron 62:593-602. CrossRef Medline

Volkow ND, Gur RC, Wang GJ, Fowler JS, Moberg PJ, Ding YS, Hitzemann R, Smith G, Logan J (1998) Association between decline in brain dopamine activity with age and cognitive and motor impairment in healthy individuals. Am J Psychiatry 155:344-349. Medline

Wang GJ, Volkow ND, Logan J, Fowler JS, Schlyer D, MacGregor RR, Hitzemann RJ, Gur RC, Wolf AP (1995) Evaluation of age-related changes in serotonin 5-HT2 and dopamine D2 receptor availability in healthy human subjects. Life Sci 56:PL249-PL253. Medline

Wong DF, Wagner HN Jr, Dannals RF, Links JM, Frost JJ, Ravert HT, Wilson AA, Rosenbaum AE, Gjedde A, Douglass KH (1984) Effects of age on dopamine and serotonin receptors measured by positron tomography in the living human brain. Science 226:1393-1396. CrossRef Medline 